\title{
Erratum: "Station for X-ray Structural Analysis of Materials and Single Crystals (Including Nanocrystals) on a Synchrotron Radiation Beam from the Wiggler at the Siberia-2 Storage Ring" [Crystallography Reports 52 (6), 1108 (2007)]
}

D. M. Kheiker ${ }^{a, b}$, M. V. Kovalchuk ${ }^{a, b}$, V. N. Korchuganov ${ }^{b}$, Yu. N. Shilin ${ }^{a}$, V. A. Shishkov ${ }^{a}$, S. N. Sulyanov ${ }^{a, b}$, P. V. Dorovatovskiï ${ }^{b, a}$, S. V. Rubinsky ${ }^{a}$, and A. A. Rusakov ${ }^{a}$

${ }^{a}$ Shubnikov Institute of Crystallography, Russian Academy of Sciences, Leninskiü pr. 59, Moscow, 119333 Russia e-mail: kheiker@nc.cryst.ras.ru

${ }^{b}$ Kurchatov Centre for Synchrotron Radiation and Nanotechnology, Russian Research Centre “Kurchatov Institute," pl. Akademika Kurchatova 1, Moscow, 123182 Russia Received March 19, 2007

PACS numbers: 99.10.Cd

DOI: $10.1134 / \mathrm{S} 1063774508020314$

On page 1108, left column:

"PACS numbers: 07.85.Qe, 07.85.Jy, 61.43.Gt, 61.10.Nz, 61.46.Df" should be read as "PACS numbers: 07.85.Qe, 07.85.Jy, 61.43.Gt, 61.10.Nz, 61.46.Df, 81.30.Dz, 81.10.Dn.”

On page 1113, Table 7, column APEX11: "0.01" should be read as "0.004."

On page 1113, Table 7, column Platinum: "0.004" should be read as "0.05." 